\title{
On the Minimization of Power Consumption in Base Stations using on/off Power Amplifiers
}

\author{
Angelos Chatzipapas \\ Institute IMDEA Networks \\ Madrid, Spain \\ Email: angelos.chatzipapas@imdea.org
}

\author{
Sara Alouf \\ INRIA Sophia Antipolis Méditerranée \\ Sophia Antipolis, France \\ Email: sara.alouf@inria.fr
}

\author{
Vincenzo Mancuso \\ Institute IMDEA Networks \\ Madrid, Spain \\ Email: vincenzo.mancuso@imdea.org
}

\begin{abstract}
Using energy generated with fossil fuel causes global warming due to the greenhouse effect, which threatens our environment. One of the challenges for New Generation Networks (NGN) is then the reduction of energy consumption, in particular at the BSs (Base Stations) which use about $85 \%$ of the total network energy. We contribute to the research with a mathematical model that calculates the total power consumption of a BS and enlightens the way to minimize it. First, we analyze the power consumed at every different component of the BS. Second, based on the cost incurred in turning off the BS's power amplifiers, we show how to decide whether it is convenient to keep the BS idle during those intervals in which no traffic has to be sent, or to turn off the amplifiers. Our model is evaluated by means of numerical examples, and shows that interesting power gain can be obtained under a large spectrum of load conditions.
\end{abstract}

\section{INTRODUCTION}

Nowadays, telecommunications, mobile Internet and many wireless applications dominate the world of Information and Communication Technology (ICT). In turn, about $2-3 \%$ of the world-wide energy consumption is for ICT, which causes about $3 \%$ of the total $\mathrm{CO}_{2}$ emissions [1]. Hence, we can understand that ICT is as useful for the human beings, as potentially harmful for our environment. The current threat to the environment could turn into a much more serious threat in the near future, e.g., due to the diffusion of mobile applications for social networks, requiring a better cellular coverage and more capabilities in the core network. The usage cost of mobile services is likely to increase, and, in particular, the energy consumption might grow with the number of BSs and data centers in the network. Hence, as the demand for ICT services rises, higher and higher energy consumption is expected for mobile radio networks.

In order to achieve lower service cost and to preserve the environment, cellular network operators try to deploy various strategies to reduce energy consumption. BSs consume about $85 \%$ of the total energy of the network. Their power consumption ranges between $\sim 147 W$ (Diet BTS 3900E [2]) and $10 \mathrm{~kW}$ depending on the size, the coverage area and the technology used [3]. The main axes of finding out efficient ways to reduce the energy consumed are: $(i)$ the optimization of hardware (which is related to hardware producers), (ii) the usage of renewable energy sources and (iii) the smart

Most of the work reported in this paper has been carried out while A. Chatzipapas and V. Mancuso were with INRIA Sophia Antipolis, France. A. Chatzipapas was also with Université de Nice, France. usage of resources through power saving models and efficient algorithms (which is related to providers).

In this paper, we present a mathematical model which illustrates the power consumption of a BS during "active" and "inactive" periods. "Active" periods are those in which the BS transmits packets while during "inactive" periods the BS stays either idle or its transmitting devices are turned off. For optimization purposes, we suggest how and when to turn the power amplifiers (PAs) of the BS off in order to reduce the power consumption. This decision depends on $(i)$ the energy cost due to switching the PAs, and $(i i)$ the power consumption incurred during idle periods.

The rest of the paper is organized as follows. Section II reports the related work and recalls existing strategies to reduce the power consumption. Section III reviews the power consumption of BS's components and further formulates a mathematical model for the total power consumption. Section IV shows how to use the model to minimize the power consumption, and finally Section $\mathrm{V}$ concludes the paper.

\section{Power SAVING STRATEgIES AND RELATED WORK}

Reducing power consumption in a cellular network, and more specifically in a BS, is possible with respect to two main constraints: $(i)$ the minimum required coverage and $(i i)$ the minimum required quality of service $(\mathrm{QoS})$ for all users.

Many operators have studied and enforced in their cellular networks new power saving mechanisms based on the inhomogeneous distribution of user traffic over time. The basic mechanism adopted is the introduction of a sleep mode, in which the BS operates at minimal power [4], [5]. When the traffic demand is scarce, most parts of the BS system are switched off and just basic functionalities are in use, like signaling from the switching center in case the system needs to power on (e.g., due to a sudden increase of traffic demand in the area). As outlined by different studies that mostly tackles the sleep mode at mobile user's side, there is a trade off between outage of users and energy saving [6]. From the operator's viewpoint, frequent switching to sleep mode may cause denial of service well beyond the operator's commitment to guarantee the availability of its services to the customers. Conversely, as shown later in our work, infrequent or no switching to sleep mode reduces the operator's opportunity to save energy.

An important characteristic of cellular networks, that is widely exploited to save energy through sleep mode-like 
operation, is the day-night behavior of the users. In fact, day activity is predominant: in the morning users are moving from residential areas to office areas while in the evening, they follow the opposite direction, leading to an aggregation of users in both areas and demanding a large capacity for both. However, when people aggregate at office areas, residential areas have light traffic needs and vice versa. During the night, both kinds of areas might experience low traffic demand. Hence, BSs with tunable capacity have been deployed, with the possibility to turn off most of the radio systems when the traffic demand is consistently low. The use of efficient software to switch off the BSs or to switch into sleep mode is a good saving technique taking advantage of the day-night behavior [7]. Most specifically, NSN [4] and NEC [8] have developed software for monitoring the traffic and automatically turning off BSs in Self Organized Networks (SONs).

Some recent papers discuss the power saving obtained by turning off underloaded BSs. In [7], the amount of energy saving is evaluated by deactivating unnecessary cells due to low traffic while in [9] a management scheme is proposed to reduce this number of unnecessary active cells. Furthermore, in [10] the cooperation between network operators could face the day-night behavior using network-wide sleeping mode and transferring the aggregated traffic to a single operator's network, sharing OPEX (operational expenses) and obtaining significant reduction of power consumption. In contrast with the existing papers, we are the first to give a complete model for the power consumption of all BS components.

On the way to green cellular networking, operators have also defined other green strategies that involve: $(i)$ selection of strategic places for deploying their network and their BSs [11], (ii) the upgrade to eco-friendly hardware, and (iii) the adoption of renewable power sources.

First, the deployment of the BSs over an area is very important for network operators. Depending on urban or rural environments with dense or light traffic the coverage strategy is different: many small BSs or a few large BSs to cover the area of interest using a single or multiple sectors per cell and one or multiple antennas (MIMO). The better the BS spatial distribution is, the less the number of BSs needed to satisfy the required coverage level and capacity, which turns into less total power consumption and less energy spent per bit [12].

Second, regarding hardware, the goal is the increased efficiency of the various BS components (rectifier, signal processing circuit, PA, feeder and cooling system). Enhancements in all parts of the BS yield a total power reduction of about $80-85 \%$ [1], [13].

Third, the adoption of renewable power sources, such as solar panels and wind generators, could play a significant role in future BSs. However, reduction of the need for fossil fuels and for connection to the electrical grid is practically possible only if BS consumption is reduced to a few hundreds of Watts [2]. Because this solution seems difficult in most cases and it has huge CAPEX (capital expenses) the use of a hybrid BS (combination of solar and diesel power generators) seems very attractive in the short term, since it obtains a reduction up to

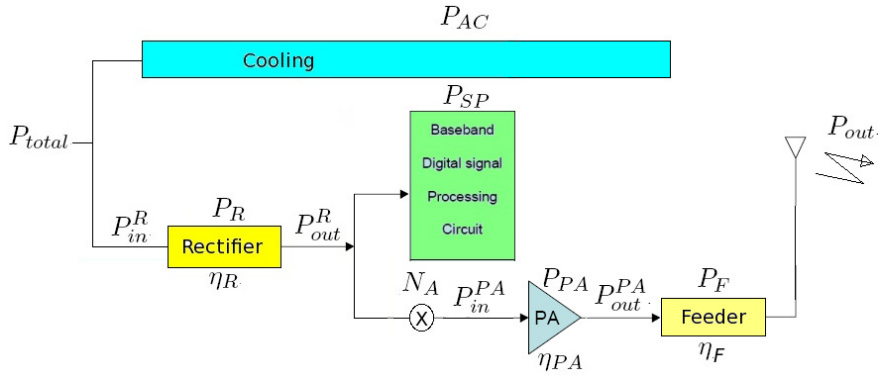

Fig. 1: Power flow in the Base Station.

$60 \%$ in energy consumption and up to $35 \%$ in CAPEX [14].

Noticeably, similarly to our work, a few number of MAC protocols have been proposed for wireless sensor networks (WSN). In particular, there are three MAC protocols, namely B-MAC [15], S-MAC [16] and T-MAC [17], where the unused sensors sleep periodically to save energy. The difficulty in adopting the philosophy of these sensor MAC protocols to the case of cellular networks, lays in the delay introduced by these MACs in the packet delivery, which is not a fundamental issue for sensor communications, while it is of paramount importance for cellular networks. Even though various energy saving solutions have been pressented in the past, we are the first to give a full representation, with a mathematical model, of a BS power consumption based on all of its components.

\section{BASE Station Model}

We will use the following notation. For a device $X$, the power consumed by $X$ is denoted $P_{X}$ and its efficiency is denoted $\eta_{X}$. The notation $P_{i n}^{X}$ and $P_{o u t}^{X}$ refers to the input and output power of the device $X . N_{A}$ denotes the number of sectors in a BS.

Fig. 1 illustrates the BS structure and the power flow. The basic components are: the rectifier, the baseband digital signal processing circuit, the PA, the feeder, the antenna and the cooling system. Next, we analyze each part of the BS and we present a mathematical model for the total power consumption.

\section{A. Energy consumption of BS components}

1) Rectifier: The rectifier transforms the signal from $\mathrm{AC}$ to DC. The efficiency of the rectifier is about $92 \%$ for a conventional rectifier and about $97 \%$ for the case of latest products, for amperage loads between 40-90\% [18]. For lower amperage loads, there are controller schemes that monitor the amperage load in order to turn off (or standby) a subset of amplifiers, so the average amperage load will increase among the rest of the amplifiers, achieving maximum efficiency [19]. The power consumption of the rectifier (dissipated as heat that needs to be removed, e.g., by the cooling system) relates with its output power $\left(P_{\text {out }}^{R}\right)$ and its efficiency $\left(\eta_{R}\right)$. It is given by:

$$
P_{R}=\frac{P_{o u t}^{R} \cdot\left(1-\eta_{R}\right)}{\eta_{R}} .
$$

2) Baseband Digital Signal Processing Circuit: The Baseband Digital Signal Processing Circuit is considered as having a constant power consumption [13]. Its approximate power 
consumption is $P_{S P}=150 \mathrm{~W}$ for conventional BSs and $P_{S P}=110 \mathrm{~W}$ for nowadays BSs. This power is dissipated as heat and has to be removed, e.g., by the cooling system.

3) Power Amplifier (PA): An amplifier is any device that magnifies the amplitude of a signal. In radio-frequency (RF) PAs, such as the one used in cellular BSs and broadcast transmitters, a very important parameter is the efficiency which is given by $\eta_{P A}=\frac{P_{\text {out }}^{P A}}{P_{P}^{P A}}$. Traditional PAs have an efficiency of about $15 \%$. The excessive energy is transformed into heat. Specialist design techniques are used to improve efficiency, such as Digital Pre-Distortion (DPD), Doherty and Envelope Tracking (ET) which can lift the efficiency up to $60 \%$ (cf., e.g., [1] and references therein).

The PA could be in four possible states: switching state, transmitting state, turned off state and idle state. During switching state, the PA commutes from active to inactive and vice versa. The probability of being in switching state $\pi_{0}$ is given by the time needed to start up, plus the time needed to switch off on average (e.g., $65 \mu s$ and $25 \mu \mathrm{s}$ respectively [20]), over the total average period $T$ between two consecutive switch-ons of the PA, $\pi_{0}=T_{s w} / T$. In transmitting state, the PA is active and boosts the signal to be transmitted over the BS air interface. The probability of being in transmitting state, $\pi_{1}$, is given by the average number of bits per packet, $S$, over the average transmission rate, $R$, times the average number of packets per second, $\lambda$. This probability is $\pi_{1}=\lambda \cdot S / R$, and represents the load of the system. The PA is in turned off state when its circuitry is inactive and no signal can be amplified. The probability of being in turned off state, $\pi_{2}$, is given by the average time $T_{\text {off }}$ of being turned off over the total average cycle duration $T$. Thus, $\pi_{2}=T_{o f f} / T$. In idle state, the PA is active but not transmitting. The probability of being in idle state is $\pi_{3}=T_{i d l e} / T$. The average power consumption for each state is $P_{s w}, P_{T_{x}}, P_{o f f}, P_{\text {idle }}$, for switching, transmission, turned off and idle powers respectively. To sum up, these are the average power consumptions computed over each cycle $T$, where each cycle $T$ is representative of the total process. The resulting model for PA's consumption is:

$$
P_{i n}^{P A}=\pi_{0} P_{s w}+\pi_{1} P_{T x}+\pi_{2} P_{o f f}+\pi_{3} P_{i d l e} \text { with } \sum_{i=0}^{3} \pi_{i}=1 .
$$

4) Feeder: The feeder is the cabling system connecting the BS to the antenna. In conventional BSs, antennas and equipments are a few meters apart, and connected through a coaxial cable. The signal attenuation of such a feeder is typically about $3 \mathrm{~dB}$. Nowadays, Remote Radio Heads (RRH) [21] technology is used instead: a very small cabinet holds the BS radio frequency devices very close to antennas, and it is connected to the (possibly remote) baseband signal processing equipment by means of optical fibers. Its efficiency is:

$$
\eta_{F}=\frac{P_{\text {out }}}{P_{\text {out }}^{P A}},
$$

and approaches 1 when using RRH, and 0.5 when using coaxial cabling. The power dissipated due to efficiencies lower than 1 is transformed into heat.

5) Cooling System: In electronic equipments and circuits, power dissipation is generally a stated condition. Electronics also have specific margins of operative temperature and in order to keep the temperature of most components of the BS within specified design limits we need to cool the sites. Air conditioners $(\mathrm{A} / \mathrm{C})$ are often the choice for radio sites. Like stated in [19], such cooling requires as much power as one third of the heat power generated inside the BS, i.e., one extra Watt is required to dissipate three Watts of heat. In addition, other cooling techniques such as free ventilation, forced-air cooling and heat exchangers have been proposed in order to save energy either for economical reasons, for energy independence of the BS, or to save battery life in off-electricgrid operated BSs. Furthermore, NSN concludes that BSs with a total power consumption less than $500 \mathrm{~W}$ (excluding the output power of the BS, $P_{\text {out }}$ ) do not use A/C system [22]. Thus, we model the power consumption at the $\mathrm{A} / \mathrm{C}$ system as:

$$
P_{A C}=\left[\frac{P_{\text {in }}^{R}-500-P_{\text {out }}}{3}\right]^{+},
$$

where, the notation $[X]^{+}=\max [X, 0]$ is used.

\section{B. Total power consumption}

Considering again Fig. 1, we observe that we have two branches forming the total power consumption of a BS: one for the cooling system and one for the power flowing into the rectifier. Thus, the total power consumption of the BS is:

$$
P_{\text {total }}=P_{\text {in }}^{R}+P_{A C}=P_{i n}^{R}+\left[\frac{P_{\text {in }}^{R}-500-P_{\text {out }}}{3}\right]^{+},
$$

with $P_{\text {out }}=\sum_{a=1}^{N_{A}} P_{\text {out }}^{(a)}$, where $N_{A}$ is the number of antennas, each transmitting with its own PA system associated. Considering multiple antennas with similar hardware implementations serving different traffic, the resulting input power at the rectifier is then as follows:

$$
\begin{aligned}
P_{i n}^{R}= & \frac{P_{S P}}{\eta_{R}}+\frac{P_{s w}}{\eta_{R}} \sum_{a=1}^{N_{A}} \pi_{0}^{(a)}+\frac{P_{T x}}{\eta_{R}} \sum_{a=1}^{N_{A}} \pi_{1}^{(a)} \\
& +\frac{P_{o f f}}{\eta_{R}} \sum_{a=1}^{N_{A}} \pi_{2}^{(a)}+\frac{P_{\text {idle }}}{\eta_{R}} \sum_{a=1}^{N_{A}} \pi_{3}^{(a)},
\end{aligned}
$$

where the superscripts within parentheses denote the specific transmitting antenna.

\section{Minimization of POWER CONSUMPtion}

In this section, we study the minimization of the total power consumption given by Eq. (5). Since we observe that, given the efficiency of rectifiers, PAs and feeder, the BS total consumption is an increasing function of the power supplied to the PA only, we show how to minimize the power consumed at the PA. Again, we have to note that the two main constraints that we take into account in order to optimize the power consumption are: $(i)$ the minimum required coverage and $(i i)$ the minimum required QoS for all users. 


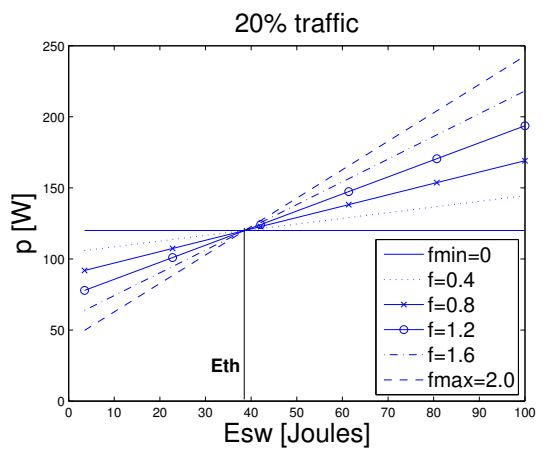

(a) $20 \%$ of traffic

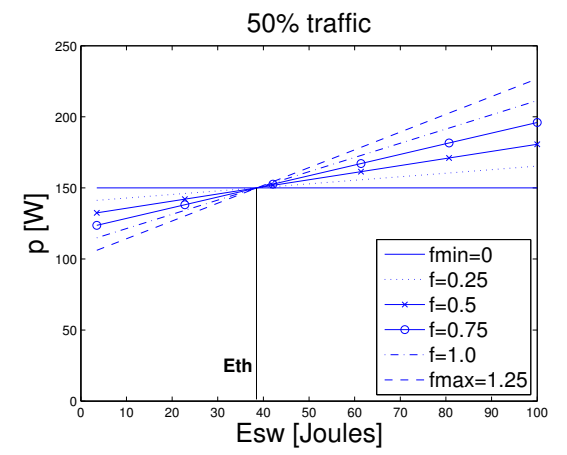

(b) $50 \%$ of traffic

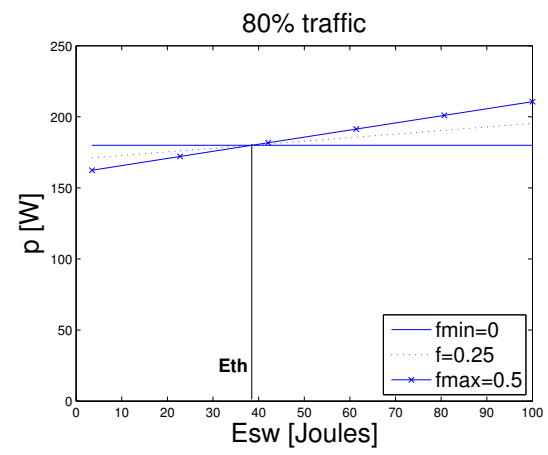

(c) $80 \%$ of traffic

Fig. 2: Power vs. switching energy for different traffic conditions and various switching frequencies.

First we define as $f=1 / T$ the average switching frequency of the PA and as $\phi$ the average fraction of time during which the PA is either idle or turned off (namely, the PA is inactive): $\phi=1-\frac{\lambda S}{R}-f T_{s w}$. When the PA is inactive, the PA is turned off with probability $\alpha$, and idle with probability $\beta=1-\alpha$. Then we reconsider the state probabilities in Eq. (2) in light of these new definitions, and considering that $(i)$ the average energy needed for switching is $E_{s w}=P_{s w} T_{s w}$, and $(i i)$ the average energy needed for one packet transmission is $E_{T x}=$ $P_{T x} \cdot \frac{S}{R}$, we rewrite Eq. (2) as follows:

$$
P_{i n}^{P A}=f \cdot E_{s w}+\lambda \cdot E_{T x}+\alpha \phi P_{o f f}+\beta \phi P_{i d l e} .
$$

Note that the fraction of time spent by the PA in turned off state, $f T_{o f f}$, and in idle state, $f T_{i d l e}$, are equal to the fractions of inactivity $\alpha \phi$ and $\beta \phi$, respectively. Thus,

$$
\alpha=\frac{f T_{\text {off }}}{1-\lambda S / R-f T_{s w}},
$$

which shows how the switching frequency $f$ and the average duration of the turned off state cannot be chosen both arbitrarily, since $\alpha \in[0,1]$. For a given traffic load, switching time and turned off state duration, the maximum switching frequency is such that $\alpha=1$, i.e.,

$$
f_{\max }=\frac{1-\frac{\lambda S}{R}}{T_{o f f}+T_{s w}} .
$$

The minimum switching frequency is always 0 , which means $\alpha=0$, i.e., never turn off the PA. Finally, note also that Eq. (7) is equivalent to the following expression for the average power consumption function $p(f)$ :

$$
\begin{aligned}
p(f)= & f\left[E_{s w}-T_{s w} P_{\text {idle }}+T_{o f f}\left(P_{o f f}-P_{\text {idle }}\right)\right] \\
& +\lambda E_{T x}+\left(1-\frac{\lambda S}{R}\right) P_{\text {idle }},
\end{aligned}
$$

which is of the form $a f+b$.

In order to find the operating point (or points) where we have the minimum power consumption we check the behavior of $a=E_{s w}-T_{s w} P_{i d l e}+T_{o f f}\left(P_{o f f}-P_{\text {idle }}\right)$.

We have to check the next three cases:

1) $a<0 \Longleftrightarrow P_{s w}<P_{\text {idle }}+\frac{T_{\text {off }}}{T_{s w}}\left(P_{\text {idle }}-P_{o f f}\right)$;

2) $a=0 \Longleftrightarrow P_{s w}=P_{\text {idle }}+\frac{T_{\text {off }}}{T_{s w}}\left(P_{\text {idle }}-P_{o f f}\right)$;
3) $a>0 \Longleftrightarrow P_{s w}>P_{\text {idle }}+\frac{T_{o f f}}{T_{s w}}\left(P_{i d l e}-P_{o f f}\right)$.

In the first case, the function decreases and the optimal value $p\left(f_{\text {opt }}\right)$ (where the power consumption is minimum) is reached at $f_{o p t}=f_{\max }$. In the second case, the coefficient $a$ is zero and the power consumption remains constant, independently of the frequency. In the last case, the function increases and thus, the optimal value $p\left(f_{\text {opt }}\right)$ is obtained when $f_{\text {opt }}=0$.

In practice, the fraction $\frac{T_{o f f}}{T_{s w}}$ is a huge value. In fact, $T_{s w}$ has a magnitude of $10^{-5^{s w}}$ seconds [20] and $T_{\text {off }}$ has a magnitude of seconds or milliseconds. Therefore, even with small values of $\left(P_{i d l e}-P_{o f f}\right)$, the occurence of cases 2$)$ and 3) requires huge values of $P_{s w}$. To show that some conditions are practically impossible to occur but theoretically possible, we use in some of the following examples and figures unreasonably high values for $P_{s w}$. However, we remark that in realistic cases, i.e., in case 1), the optimal frequency of the system is $f_{\text {opt }}=f_{\max }$.

In Fig. 2 we represent the power consumed at the PA with respect to the switching energy for various switching frequencies. Fig. 2a corresponds to $20 \%$ of traffic load $\left(\frac{\lambda S}{R}=0.2\right)$, Fig. $2 \mathrm{~b}$ to $50 \%$ of load $\left(\frac{\lambda S}{R}=0.5\right)$, and Fig. $2 \mathrm{c}$ to $80 \%$ of load $\left(\frac{\lambda S}{R}=0.8\right)$. The values that we used in Eq. (10) to plot these graphs are as follows. As for the load, we use $\lambda \in\{2,5,8\} \mathrm{pkt} / \mathrm{s}, S=100 \mathrm{bits}$, and $R=1000 \mathrm{bits} / \mathrm{s}$, but notice that all quantity expressing power consumption, including the term $\lambda E_{T x}$ in Eq. (10), depend only on the load $\frac{\lambda S}{R}$, and not on the particular value of $\lambda, S$, and $R$. As for the time and consumption in the different PA states, we use realistic values [20]: $T_{s w}=0.00009 \mathrm{~s}, E_{T x}=20 \mathrm{~J}$ (corresponding to an output power of $20 \mathrm{~W}$ with $\eta_{P A}=0.2$, $\eta_{F}=0.5$, and $\left.E_{T x}=\frac{20 \cdot S}{\eta_{P A} \eta_{F} R}\right), P_{\text {idle }}=100 \mathrm{~W}, P_{\text {off }}=$ $3.5 \mathrm{~W}, T_{\text {off }}=0.4 \mathrm{~s}$. So, the power consumption when the PA transmits a packet is about $200 \mathrm{~W}$, when it is in idle state, $100 \mathrm{~W}$ and when it is in turned off state, $3.5 \mathrm{~W}$.

In Fig. 2, power curves obtained at different switching frequencies $f$ intersect at $E_{s w}=E_{t h}=T_{s w} P_{\text {idle }}-$ $T_{\text {off }}\left(P_{\text {off }}-P_{\text {idle }}\right)$ and thus $E_{t h}=38.69 \mathrm{~J}$, independently of the traffic load. In the figure, values of $E_{s w}$ smaller of $E_{t h}$ correspond to case 1), $E_{s w}=E_{t h}$ corresponds to case 2), and $E_{s w}>E_{t h}$ corresponds to case 3$)$. Therefore, considering that the switching power is $P_{s w}=E_{s w} / T_{s w}$, with $T_{s w}$ very small, 


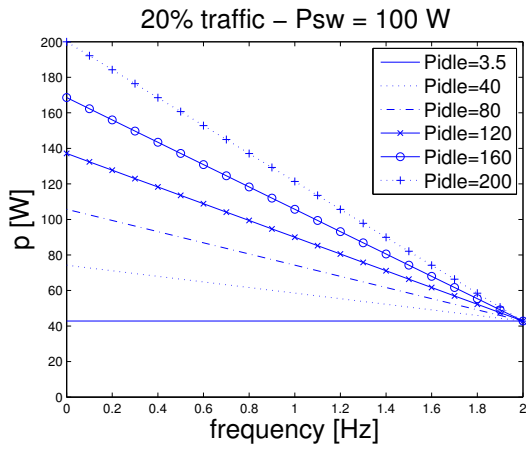

(a) $20 \%$ of traffic

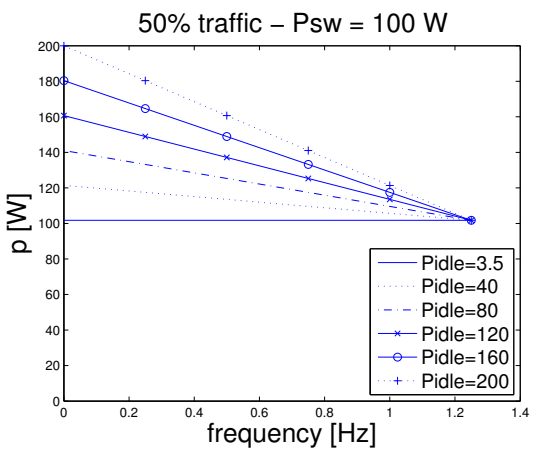

(b) $50 \%$ of traffic

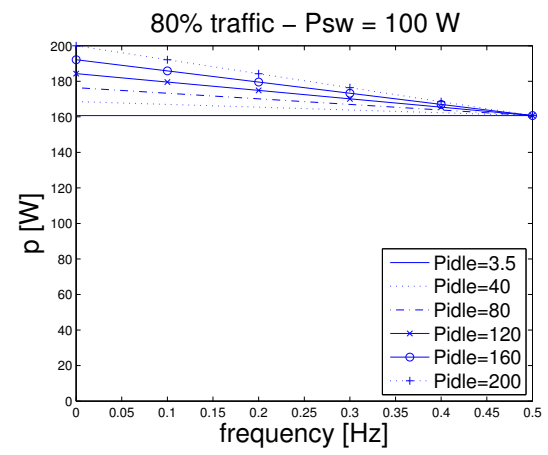

(c) $80 \%$ of traffic

Fig. 3: Power vs. switching frequency, for various traffic conditions, different $P_{i d l e}$ and $P_{s w}=100 \mathrm{~W}$.

$P_{s w}$ has to be very large (tens of thousands of Watts) in order to fall in case 2) or 3). We can conclude that $E_{s w}$ remains to the left side of the intersection for every realistic value of $P_{s w}$, for which we observe that the minimum consumption is obtained when $f=f_{\max }$.

In Fig. 3, we illustrate the power consumption at different frequencies for various values of $P_{\text {idle }}$ and different traffic conditions. For this figure, the values we used in Eq. (10) are the same as before; additionaly we fixed $E_{s w}=0.009 \mathrm{~J}$ $\left(P_{s w}=1000 \mathrm{~W}\right)$. From the figure, one can observe that the smaller $P_{\text {idle }}$ is, the less interesting it is to turn off the PA.

Gain. To compute the gain obtained by turning off the PA, we compare the power consumption of a PA that is never turned off, thus given by $p(0)$, to the one achieved at some switching frequency, $p(f)$. So, the absolute gain is:

$$
\begin{aligned}
G(f) & =p(0)-p(f) \\
& =f \cdot\left[P_{i d l e}\left(T_{s w}+T_{o f f}\right)-\left(T_{s w} P_{s w}+T_{o f f} P_{o f f}\right)\right],
\end{aligned}
$$

and the relative gain is $G(f) / p(0)$. Note that the absolute gain does not directly depend on the traffic load, even though it depends on $f$, whose optimal value can be a function of the load-i.e., $f_{\max }$ in case 1 ).

In Fig. 4a and Fig. 4b we show the absolute gain as a function of $P_{\text {idle }}$ and $f$, for high values of $P_{s w}$. By comparing the two figures, it is clear that using $f=0$ is convenient only below a $P_{\text {idle }}$ threshold, and that this threshold grows with $P_{s w}$. However, thresholds reasonably higher than a few Watts can be obtained only with unreasonably high values of the switching power $P_{s w}$ (several tens of $k W$ ).

In Figs. 5 and 6 we plot the relative gain we obtain by switching off the PA for $P_{s w}=1000 \mathrm{~W}$ and $100000 \mathrm{~W}$. We observe that the higher the $P_{\text {idle }}$ of the PA, the higher the gain by switching off more frequently. We see that in cases where $P_{\text {idle }}$ is close to the transmission power we can gain almost $80 \%$ of power by switching more frequently. For both cases there is an intersection point at $P_{\text {idle }}=\frac{T_{o f f} P_{o f f}+T_{s w} P_{s w}}{T_{o f f}+T_{s w}}$. In Fig. 5 where $P_{s w}=1000 \mathrm{~W}$ this value is $P_{\text {idle }}=3.724 \mathrm{~W}$ and in Fig. 6 where $P_{s w}=100000 \mathrm{~W}$, it is $P_{\text {idle }}=25.994 \mathrm{~W}$. For greater values of $P_{\text {idle }}, a<0$, and $f_{\text {opt }}=f_{\max }$, while for smaller values, $a>0$ and $f_{\text {opt }}=0$ meaning that for $P_{\text {idle }}$ close to $P_{o f f}$, it is optimal to stay idle. Note that the gain

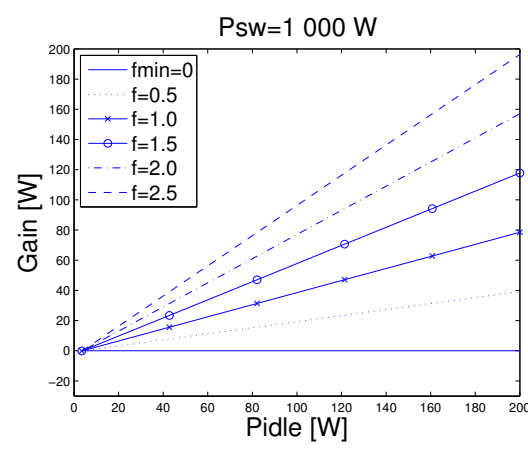

(a) Gain with $P_{s w}=1000 \mathrm{~W}$ (high value). With $P_{i d l e}$ larger than a few Watts, the gain grown with the PA switching frequency $f$.

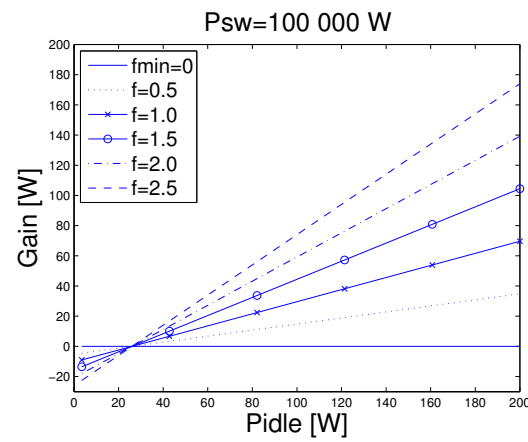

(b) Gain with $P_{s w}=100000 \mathrm{~W}$ (unreasonably high). With reasonably low values of $P_{\text {idle }}$ (few tens of Watts) it is not convenient to turn off the PAs.

Fig. 4: Gain vs. $P_{\text {idle. }}$ The maximum achievable switching frequency is limited by the traffic load, i.e., $f \leq \frac{1-\frac{\lambda S}{R}}{T_{o f f}+T_{s w}}$.

depicted in the figures is a conservative estimate because we show results for high values of $P_{s w}$.

Furthermore, we have to point that the maximum frequency changes due to the limitation on the upper bound for $f_{\max }$. So, as soon as the load $\frac{\lambda S}{R}$ increases, the maximum frequency decreases, as well as the maximum gain decreases..

\section{CONClusion}

This paper presented a mathematical model to compute and minimize the base station power consumption. The gain achieved by reducing the power consumption is twofold. First, $\mathrm{CO}_{2}$ emissions can be dramatically reduced, therefore 


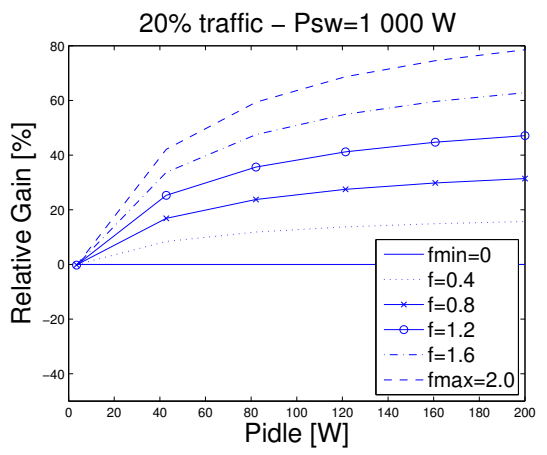

(a) $20 \%$ of traffic

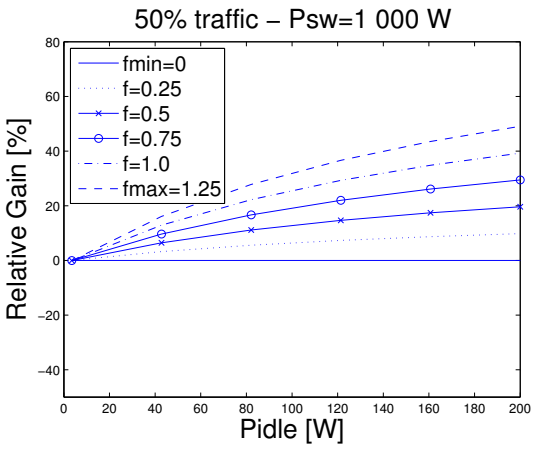

(b) $50 \%$ of traffic

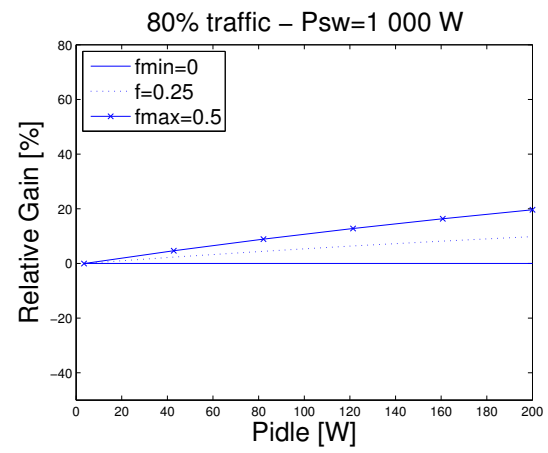

(c) $80 \%$ of traffic

Fig. 5: Relative Gain vs. $P_{i d l e}$ for different switching frequencies, different traffic conditions and $P_{s w}=1000 \mathrm{~W}$.

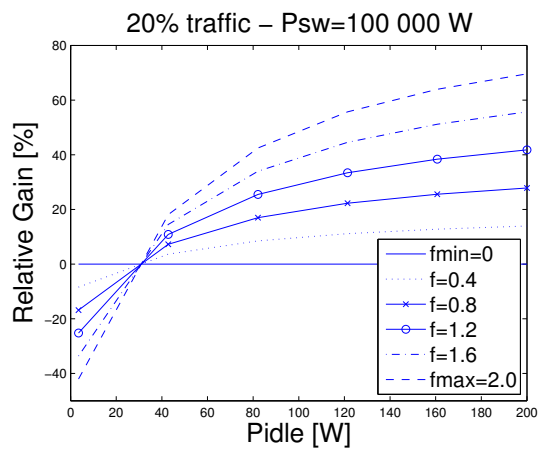

(a) $20 \%$ of traffic

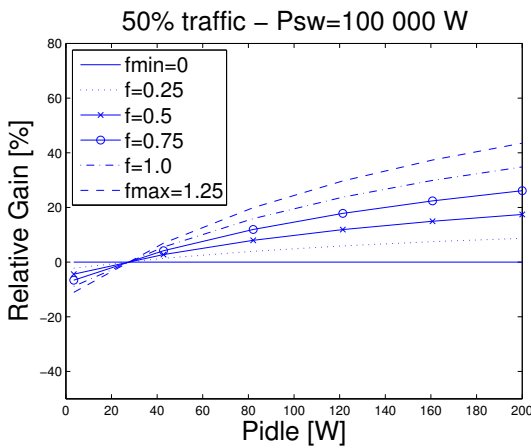

(b) $50 \%$ of traffic

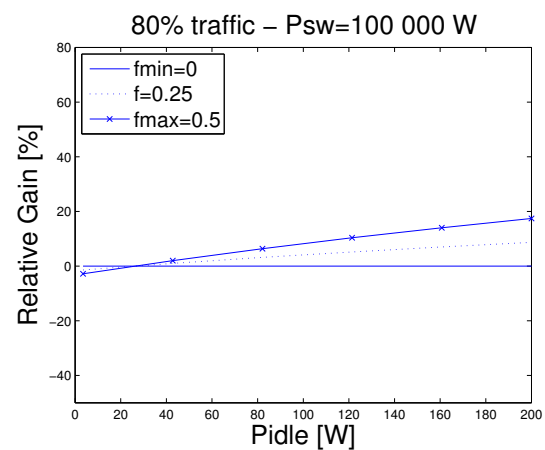

(c) $80 \%$ of traffic

Fig. 6: Relative Gain vs. $P_{i d l e}$ for different switching frequencies, different traffic conditions and $P_{s w}=100000 \mathrm{~W}$.

preserving the environment. Second, the operational expenses of cellular providers, and hence the service cost for data exchanges, can be made much cheaper and affordable.

Our model accounts for all main components of a base station, whose costs are combined in order to have a complete and reliable cost model. We showed that turning off the power amplifiers is often more convenient than keeping them idle, but the switching frequency needs to be evaluated as a function of the cell load. Noticeably, even for high traffic conditions, it is possible to save up to $20 \%$ of power, while under low to and medium traffic conditions the gain can rise up to $80 \%$.

\section{REFERENCES}

[1] V. Mancuso, S. Alouf, Reducing costs and pollution in cellular networks, IEEE Communications Magazine, special issue on Energy Efficiency in Communications: Part III, August 2011.

[2] Vodafone, Vodafone Green Technology Demo, MWC 2010, http://www.vodafone.com/content/dam/vodafone/about/sustainability/ green\%20technology\%20demo\%20MWC\%202010_PUBLIC.pps.

[3] A. Hikuma, Y. Fuke, N. Nakaninami, H. Ohyane, H. Kobayashi, Radio Base Stations Equipments toward Economical Expansion of FOMA Coverage Areas, NTT DoCoMo. Technical Journal, Jun. 2004.

[4] NSN, Introducing the Nokia Siemens Networks SON Suite - An efficient, future proof platform for SON, white paper, Nov. 2009.

[5] NEC, NEC's approach towards Energy-efficient Radio Access Networks, white paper, Feb. 2010.

[6] S. Alouf, E. Altman, and A. Prakash Azad, M/G/1 Queue with Repeated Inhomogeneous Vacations Applied to IEEE 802.16e Power Saving, ACM Sigmetrics 2008.

[7] M. Ajmone-Marsan, L. Chiaraviglio, D. Ciullo, M. Meo, Optimal Energy Savings in Cellular Access networks, GreenComm 2009, Dresden.

[8] NEC, Self Organizing Network: NEC's proposals for next-generation radio network management, white paper, Feb. 2009, http://www.nec.com/ global/solutions/nsp/lte/pdf/son.pdf.
[9] L. Chiaraviglio, D. Ciullo, M. Meo, M. Ajmone-Marsan, Energy-Efficient Management of UMTS Access Networks, W-GREEN 2008, Lapland.

[10] M. Ajmone-Marsan, M. Meo, Energy efficient management of two cellular access networks, GreenMetrics 2009 Workshop.

[11] P. Herhold, W. Rave, G. Fettweis, Relaying in CDMA Networks: Pathloss Reduction and Transmit Power Savings, 57th Vehic. Technol. Conf. Korea, April 2003.

[12] M. Etoh, T. Ohya, Y. Nakayama, Energy Consumption Issues on Mobile Network Systems, IEEE SAINT 2008, Turku, Finland, July 2008.

[13] Alcatel-Lucent, How to reduce green house gas emissions from ICT equipment, ETSI Green Agenda, Nov. 2009.

[14] Motorola, The power play: Reducing the build and power consumption costs of WiMAX base stations, white paper, June 2009, http: //www.motorola.com/staticfiles/Business/Solutions/IndustrySolutions/ ServiceProviders/NetworkOperators/LTE/_Document/LTE_Documents/ Static_Files/WiMAXCivilSiteWP-FINAL.pdf?localeId=33.

[15] J. Polastre, J. Hill, D. Culler, Versatile low power media access for wireless sensor networks, SenSys '04, Nov. 2004.

[16] W. Ye, J. Heidemann, D. Estrin, Medium access control with coordinated, adaptive sleeping for wireless sensor networks, in IEEE Transactions on Networking, Apr. 2004.

[17] T. van Dam, K. Langendoen, An adaptive energy-efficient MAC protocol for wireless sensor networks, in Proceedings of the First ACM Conference on Embedded Networked Sensor Systems, Nov. 2003.

[18] Emerson Electric, Rectifier Efficiency, http://www.emerson.com/sites/ Network_Power/en-US/Products/GenericProduct/Pages/near97percent. aspx?8109C $=$ Greece.

[19] S. Roy, Energy Logic: A Road Map to reducing Energy Consumption in Telecommunication Networks, Proceedings of INTELEC, 2008.

[20] Nujira, coolteq.h NCT-H4010, High Voltage Modulator - Preliminary Datasheet, 2009.

[21] C. Lanzani, G. Kardaras, D. Boppana Remote Radio Heads and the evolution towards $4 G$ networks, white paper, Feb. 2009, http://www.altera. com/literature/wp/wp-01096-rrh-4g.pdf

[22] NSN, Infrastructure Asia, http://www.infrastructureasia.com/ speakers100416/100416-asiapasificparallel2renewable-paulyap-nsn.pdf. 